УДК 004.051

\title{
АЛГОРИТМ И ПРОГРАММА ОПТИМАЛЬНОГО РЕЗЕРВИРОВАНИЯ ФУНКЦИОНАЛЬНЫХ УЗЛОВ ИНТЕГРИРОВАННЫХ СИСТЕМ БЕЗОПАСНОСТИ
}

\author{
В. А. Дурденко , Э. К.Алгазинов ${ }^{\star}$ А. А. Рогожин ${ }^{\star *}$, Д. С. Богданов ${ }^{\star *}$, Б. О. Баторов ${ }^{\star * \star}$ \\ ${ }^{*}$ Воронежский государственный университет \\ ${ }^{*}$ Краснодарский университет МВД России \\ ${ }_{* * \star}$ Академия управления МВД России
}

Поступила в редакцию 16.07.19 г.

\begin{abstract}
Аннотация. В случаях, когда на объектах, подлежащих обязательной государственной охране, необходима модернизация или повышение надежности/эффективности интегрированных систем безопасности (ИСБ), то рассматривается вопрос оптимального резервирования их функциональных узлов (функционально необходимых элементов). Тогда возможна постановка двух следующих задач, связанных с построением оптимальной структуры ИСБ при резервировании:

1. Требуется обеспечить надежность или эффективность ИСБ не менее заданной при минимальных затратах на резервные элементы.

2. Требуется обеспечить максимально возможную надежность или эффективность ИСБ при заданных затратах на резервные элементы.

Для решения задач оптимального резервирования ИСБ является метод покоординатного спуска, который относится к поисковым методам решения задач нелинейного программирования, позволяющим получить приближенное решение с некоторой заданной точностью. Предложены алгоритм и программа оптимального резервирования функциональных узлов ИСБ с целью повышения эффективности и надежности их функционирования. В основу разработанного алгоритма заложен метод покоординатного спуска. Программа работает в двух режимах: в режиме оптимального расчета стоимости ИСБ и в режиме расчета необходимой эффективности ИСБ при минимальных затратах на резервирование. Программа позволяет загружать и обрабатывать более $2^{15}$ строк (элементов) исходных данных. Ключевые слова: эффективность, надежность, оптимальное резервирование, функциональный узел, интегрированная система безопасности.
\end{abstract}

\section{ВВЕДЕНИЕ}

В настоящее время обеспечение безопасности объектов, подлежащих обязательной государственной охране, уполномочены обеспечивать профессионально подготовленные и соответствующим образом оснащенные подразделения вневедомственной охраны войск национальной гвардии Российской Федерации, ФГУП «Охрана» Росгвардии, фе-

(ㄷ Дурденко В. А., Алгазинов Э. К., Рогожин А. А., Богданов Д. С., Баторов Б. О., 2019 деральной службы охраны и ведомственной охраны федеральных государственных органов, в том числе с помощью современных интегрированных систем безопасности $[4,6]$.

В случаях, когда на объектах, подлежащих обязательной государственной охране, необходима модернизация или повышение надежности/эффективности ИСБ, то рассматривается вопрос оптимального резервирования их функциональных узлов (функционально необходимых элементов). Тогда возможна постановка двух следующих задач, связанных с построением оптимальной структуры ИСБ 
при резервировании $[1,5]$ :

1. Требуется обеспечить надежность или эффективность ИСБ не менее заданной при минимальных затратах на резервные элементы.

2. Требуется обеспечить максимально возможную надежность или эффективность ИСБ при заданных затратах на резервные элементы.

Первая задача может быть сформулирована в следующей форме - найти минимум функции:

$$
W=\sum_{i=1}^{n}\left(w_{i} m_{i}+w_{i}^{0}\right)
$$

при условии:

$$
P=\prod_{i=1}^{n} p_{i}\left(m_{i}\right) \geq P_{m p} .
$$

Вторая задача формулируется так - найти максимум функции:

$$
P=\prod_{i=1}^{n} p_{i}\left(m_{i}\right)
$$

при условии:

$$
W=\sum_{i=1}^{n}\left(w_{i} m_{i}+w_{i}^{0}\right) \leq W_{m p}
$$

где $P$ - вероятностный показатель надежности (эффективности) ИСБ;

$P_{m p}$ - требуемое значение вероятностного показателя надежности (эффективности) ИСБ;

$W$ - стоимость ИСБ;

$W_{m p}$ - требуемое значение стоимости ИСБ;

$m$ - число резервных элементов на $i$-м участке ИСБ;

$n$ - число различных участков ИСБ;

$w_{i}$ - стоимость одного элемента $i$-го участка ИСБ;

$p_{i}\left(m_{i}\right)$ - вероятностный показатель надежности (эффективности) $i$-го участка ИСБ при наличии на нем $m$ резервных элементов;

$w$ - начальная стоимость $i$-го участка ИСБ.

\section{МАТЕРИАЛЫ И МЕТОДЫ}

Учитывая тот факт, что ИСБ являются достаточно сложными организационно-техническими системами, число элементов ИСБ может достигать десятки и сотни тысяч в зависимости от сложности и масштаба охраняемого объекта. В этой связи может возник- нуть ситуация, когда и число резервируемых участков должно быть достаточно большим (больше 1000). В этом случае одним из наиболее удобных численных методов решения задач оптимального резервирования является метод покоординатного спуска $[3,7,8]$, который относится к поисковым методам решения задач нелинейного программирования, позволяющим получить приближенное решение с некоторой заданной точностью. Достоинством данного метода является обеспечение наименьшего числа шагов решения задачи поиска.

Моделирование оптимальной резервированной ИСБ можно представить в виде следующего многошагового процесса [1].

Рассматривается некоторая исходная ИСБ, у которой на каждом участке не имеется сначала ни одного резервного элемента.

На первом шаге отыскивается такой участок системы, прибавление к которому одного резервного элемента дает наибольший «удельный» выигрыш в приросте показателя надежности или эффективности.

На втором шаге отыскивается следующий участок в системе (включая и тот, у которого уже есть резервный элемент), который характеризуется наибольшим приростом показателя надежности или эффективности при добавлении одного резервного элемента.

Аналогичным образом процесс продолжается далее.

Допускается, что каждый $i$-й участок ИСБ уже зарезервирован $m_{i}$ раз, т. е. всего уже сделано $M=\sum^{n} m_{i}$ шагов описанного процесса.

Значеніие вероятности безотказной работы системы на $M$-м шаге процесса равно:

$$
P^{(M)}=P\left(m_{i}, m_{2}, . ., m_{n}\right)=\prod_{i=1}^{n} p_{i}\left(m_{i}\right) .
$$

В соответствии с принятым алгоритмом на следующем, $(M+1)$-м, шаге процесса следует добавить еще один резервный элемент на такой участок системы, для которого максимальной является величина:

$$
\begin{gathered}
\gamma_{i}\left(m_{i}+1\right)=\frac{1}{w_{i}}\left[P\left(m_{i}, m_{2}, . ., m_{i}+1, . ., m_{n}\right)-\right. \\
\left.-P\left(m_{i}, m_{2}, . ., m_{i}, . ., m_{n}\right)\right] .
\end{gathered}
$$




\section{В. А. Дурденко, Э. К.Алгазинов, А. А. Рогожин, Д. С. Богданов, Б. О. Баторов}

Выражая $P^{(M)}$ и $P^{(M+1)}$ через вероятности безотказной работы отдельных участков системы, получаем значение $\gamma_{i}\left(m_{i}+1\right)$ в виде:

$$
\begin{gathered}
\gamma_{i}\left(m_{i}+1\right)= \\
=\frac{1}{w_{i}}\left[p_{i}\left(m_{i}+1\right) \prod_{\substack{k=1 \\
k \neq 1}}^{n} p_{k}\left(m_{k}\right)-\prod_{k=1}^{n} p_{k}\left(m_{k}\right)\right]= \\
=\frac{1}{w_{i}} \prod_{k=1}^{n} p_{k}\left(m_{k}\right)\left[\frac{p_{i}\left(m_{i}+1\right)}{p_{i}\left(m_{i}\right)}-1\right]= \\
=\frac{p_{i}\left(m_{i}+1\right)-p_{i}\left(m_{i}\right)}{w_{i} p_{i}\left(m_{i}\right)} .
\end{gathered}
$$

Величина $P^{(M)}$ входит в выражения значений $\gamma_{i}\left(m_{i}+1\right)$ для всех $i=1,2, \ldots, n$. Поскольку на каждом шаге процесса интересует только относительная величина $\gamma_{i}\left(m_{i}+1\right)$ по сравнению с другими такими же величинами, протекание процесса не изменится, если на каждом шаге выбирать тот участок, у которого наибольшей является величина:

$$
\gamma_{i}\left(m_{i}+1\right)=\frac{p_{i}\left(m_{i}+1\right)-p_{i}\left(m_{i}\right)}{w_{i} p_{i}\left(m_{i}\right)} .
$$

Рассчитав для каждого участка ИСБ значения $\gamma_{i}\left(m_{i}+1\right)$, можно определить, в какой последовательности следует добавлять резервные элементы, чтобы, остановившись на любом произвольном шаге процесса, получалась система, резерв которой оптимален.

С учетом вышеизложенного в целях совершенствования системы управления безопасностью объектов, подлежащих обязательной государственной охране и оборудованных ИСБ, разработаны соответствующие алгоритм и программа оптимального резервирования функциональных узлов ИСБ с целью повышения эффективности и надежности их функционирования [2].

Укрупненная блок-схема разработанного алгоритма оптимального резервирования функциональных узлов ИСБ с целью повышения эффективности и надежности их функционирования приведена на рис. 1.

В блок-схеме разработанного алгоритма оптимального резервирования функциональных узлов ИСБ использованы следующие условные обозначения:
- double $\mathrm{x}[\mathrm{n}]$ - массив «х», состоящий из «n» элементов;

- ofd_input - класс вызова диалогового окна;

- int $\mathrm{x}=0$ - объявление переменной «х»;

- $\mathrm{x}=$ true - возвращает true, если условие истинно;

- \&\& - логическое «И»;

- $\mathrm{i}=0$... $\mathrm{x}$ - цикл с инкрементом «i» от 0 до $\mathrm{x}$;

- ${ }^{* *} \mathrm{y}[\mathrm{i}]$ - возведение «х» в степень «i» элемента числового массива «у»;

- $\mathrm{x}++$ - инкремент переменной;

- return x - возвращение значения переменной «х» после выполнения вычислений;

- $\max (\mathrm{x}[])$ - метод нахождения максимального значения в массиве.

\section{РЕЗУЛЬТАТЫ И ИХ ОБСУЖДЕНИЕ}

На основе предложенного алгоритма разработана программа для ЭВМ, которая предназначена для автоматизированного расчета оптимального количества резервных элементов в ИСБ. Учитывая, что в основу алгоритма программы заложен численный метод покоординатного спуска, это позволило минимизировать время, затраченное на расчеты и увеличить их точность.

Программа работает в двух режимах: в режиме оптимального расчета стоимости ИСБ и в режиме расчета необходимой эффективности ИСБ при минимальных затратах на резервирование.

Минимальные требования к ЭВМ для работы программы:

- процессор: Pentium 4 / Athlon 642 ГГц;

- память: 256 МБ;

- видеокарта: 256 МБ;

- свободное место на ЖД: 10 МБ.

Программа написана с использованием языка Visual C\#.

Вид и версия операционной системы: Microsoft Windows 7, 8, 8.1, 10.

Объем программы для ЭВМ в машиночитаемой форме: 2,68 Мб.

Основные окна интерфейса программы оптимального резервирования функциональных узлов ИСБ объектов, подлежащих обязательной государственной охране, представлены на рис. 2, 3. 
Алгоритм и программа оптимального резервирования ...

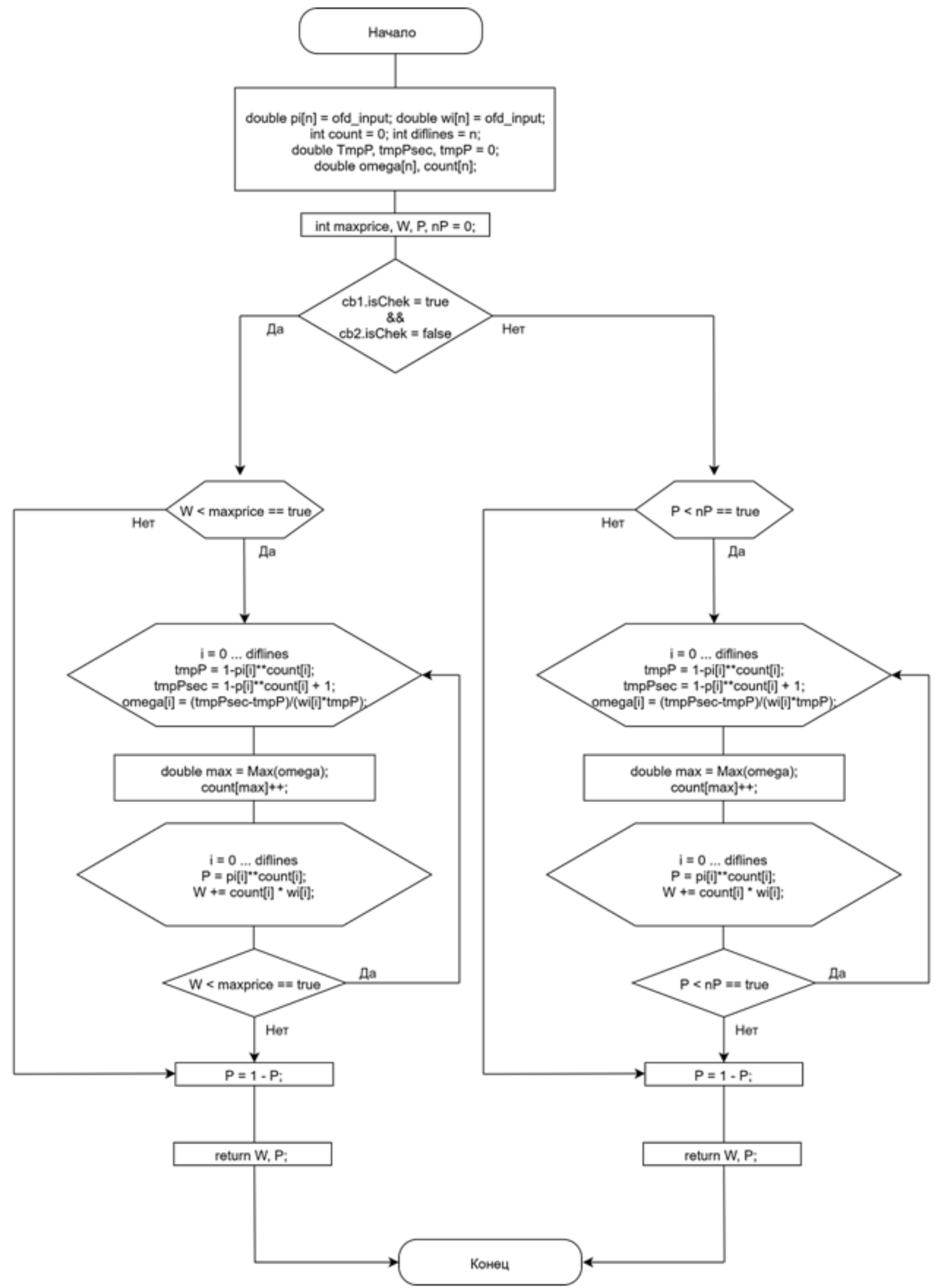

Рис. 1. Блок-схема разработанного алгоритма оптимального резервирования функииональных узлов ИСБ 


\section{Загрузить данные}

Oптимальная стоимость

\section{0}

Оптимальная надежность

Эффектиеность / надекность: 0,999994912921842

Стоимость ИСЕ: 5700

Bcero резервных участков: 3

\section{Oиnстить}

Bыnолнить расчет

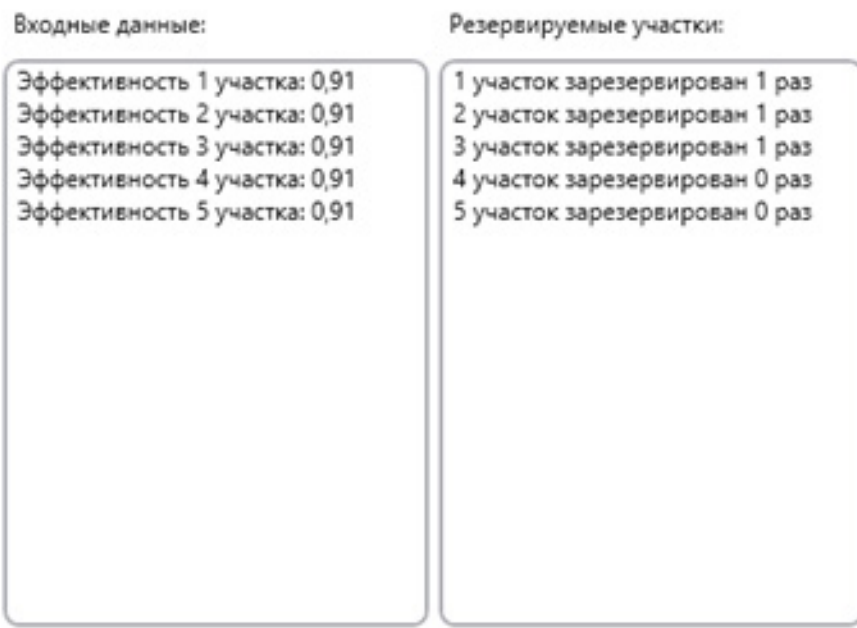

Рис. 2. Окно программы после расчета оптимального резервирования ИСБ по критерию стоимости

Загрузить Аанные

Oптимальная стоимость

Oптимальная надекность

0,999999

Эффективмость / надекмость: 0,999999070528344

Стоимость ИСБ: 1415500

Bcero pesepentix участков: 745

\section{Очистить}

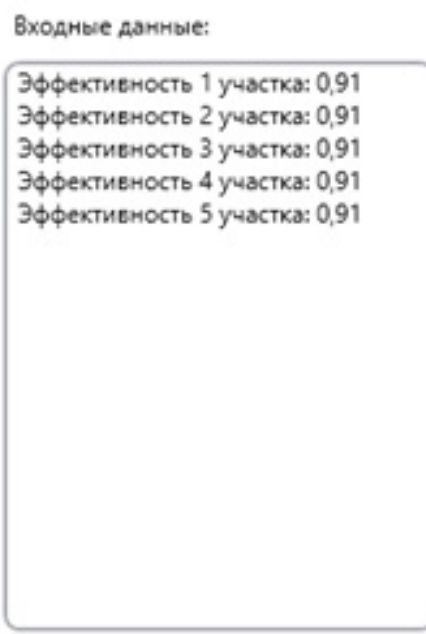

Pеsереируемые участки:

1 участок зарезереироезт 149 раs 2 участок зарезервирован 149 раз 3 участок зарезереироеам 149 раз 4 участок зарезереирован 149 раз

5 участок зарезервировам 149 раз

Рис. 3. Окно программы после расчета оптимального резервирования ИСБ по критерию эффективности

Краткая инструкция по использованию программы оптимального резервирования функциональных узлов ИСБ объектов, подлежащих обязательной государственной охране:

- при запуске программы необходимо выбрать критерий оптимального резервирования, установив в соответствующем месте галочку. При выборе оптимальной стоимости указывается стоимость в условных единицах, при выборе оптимальной эффективности
ИСБ в поле указывается требуемая эффективность от 0 до 1.

- После выбора и установления требуемого критерия осуществляется импорт исходных данных из файла с расширением *.txt при нажатии на кнопку «Загрузить данные». Формат заполнения файла с исходными данными: «0,95;0,94;..;n|1000;900;...;n» построчно, где каждая строка соответствует резервируемому участку, который включает в себя n элементов. Данные, указанные до символа «|» 
соответствуют вероятности безотказной работы элементов, коэффициенту готовности (вероятности реализации элементом своей целевой функции), а после - цены на соответствующие элементы в у. е. При этом, например, на 1 участке может быть n резервных элементов, а на 2 участке $\mathrm{m}$.

- После импорта данных в правой части программы в элементе вывода «Входные данные» отображается информация о загруженной информации. При нажатии на кнопку «Выполнить расчет» осуществляется вычисление оптимального резерва ИСБ в соответствии с условиями, заданными на шаге 1.

- По окончании расчетов в левой нижней части окна программы выводятся результаты выполнения, которые включат в себя «Эффективность / надежность ИСБ» И «Стоимость ИСБ». Так же в правой части окна программы в элементе вывода «Резервируемые участки» отображаются все участки системы и соответствующее им количество резервирований.

- При нажатии на кнопку «Очистить» осуществляется очистка всех переменных, массивов и элементов вывода, включая импортированные данные.

- Программа позволяет загружать и обрабатывать более $2^{15}$ строк (элементов) исходных данных.

\section{ЗАКЛЮЧЕНИЕ}

В статье в целях совершенствования системы управления безопасностью объектов, подлежащих обязательной государственной охране и оборудованных ИСБ, разработаны алгоритм и программа оптимального резервирования функциональных узлов интегрированных систем безопасности объектов, подлежащих обязательной государственной охране, отличающиеся использованием численного метода покоординатного спуска, что позволяет минимизировать время, затраченное на проведение вычислительного эксперимента, и возможностью одновременного решения двух задач:

1. Обеспечить надежность или эффективность ИСБ не менее заданной при минимальных затратах на резервные элементы.
2. Обеспечить максимально возможную надежность или эффективность ИСБ при заданных затратах на резервные элементы.

Область применения разработанной программы может распространяться на решение подобных задач оптимального резервирования других сложных организационно-технических систем.

\section{СПИСОК ЛИТЕРАТУРЫ}

1. Дурденко, В. А. К вопросу об оптимальном резервировании функциональных узлов интегрированных систем безопасности объектов, подлежащих обязательной государственной охране / В. А. Дурденко, А. А. Рогожин, Б. О. Баторов // Информатика: проблемы, методология, технологии: материалы XVIII международной научно-методической конференции. Том 7. - Воронеж : Воронежский государственный университет, 2018. C. 151-156.

2. Баторов, Б. О. Программа оптимального резервирования функциональных узлов интегрированных систем безопасности объектов, подлежащих обязательной государственной охране / Б. О. Баторов, А. А. Рогожин, Д. С. Богданов. - Москва : Федеральная служба по интеллектуальной собственности, 2018. - Свидетельство о государственной регистрации программы для ЭВМ от 19.06.2018 № 2018617151 .

3. Надежность технических систем : учебное пособие для вузов технических специальностей / под общ. ред. Е. В. Сугака и Н. В. Василенко. - Красноярск : НИИ СУВПТ, 2001. $608 \mathrm{c}$.

4. Рогожин, А. А. Основы построения интегрированных систем безопасности : учеб. пособие / А. А. Рогожин. - Воронеж : Воронежский институт МВД России, 2012. - 74 с.

5. Рогожин, А. А. Разработка методики построения оптимальной надежной структуры подсистемы охранно-пожарной сигнализации интегрированной системы безопасности на объекте информатизации / А. А. Рогожин // Научная сессия ТУСУР-2012: электронные материалы XIII Всероссийской 
научно-технической конференции. Том 3. Томск : ТУСУР, 2012. - С. 215-218.

6. Рогожин, А. А. Тактика применения интегрированных систем безопасности : учеб. пособие / А. А. Рогожин, А. В. Эсауленко. Воронеж : Воронежский институт МВД России, 2014. - 278 с.
7. Ромашова, О. Ю. Методы оптимизации и расчеты на ЭВМ технико-экономических задач: учебное пособие / О. Ю. Ромашова. Томск : Изд-во ТПУ, 2009. - 210 с.

8. Ушаков, И. А. Курс теории надежности систем / И. А. Ушаков. - Москва : Дрофа, 2008. - 238 c.

Дурденко Владимир Андреевич - д-р техн. наук, профессор кафедры информационных технологий управления, Воронежский государственный университет.

E-mail:dva_viis@mail.ru

Алгазинов Эдуард Константинович - д-р физ.-мат. наук, проф., декан факультета компьютерных наук, Воронежский государственный университет.

E-mail: algazinov@sc.vsu.ru

Рогожин Александр Александрович - канд. техн. наук, заместитель начальника отдела организации учебного процесса, Краснодарский университет МВД России.

E-mail: raa_tsbs@list.ru.

Богданов Дмитрий Сергеевич - курсант, Краснодарский университет МВД России.

E-mail: bdkrdu@yandex.ru.

Баторов Батор Октябрьевич - адъюнкт кафедры информационных технологий, Академия управления МВД России.

E-mail: 03bator@rambler.ru 


\title{
THE ALGORITHM AND PROGRAM FOR OPTIMAL REDUNDANCY OF FUNCTIONAL UNITS OF THE INTEGRATED SECURITY SYSTEMS
}

\author{
V. A. Durdenko ${ }^{\star}$, E. K. Algazinov ${ }^{\star}$ A. A. Rogozhin ${ }^{\star \star}$, D. S. Bogdanov ${ }^{\star \star}$, B. O. Batorov ${ }^{\star \star \star}$ \\ ${ }^{*}$ Voronezh State University \\ ${ }^{*}$ Krasnodar university of the Ministry of the Interior of Russia \\ ${ }^{* * *}$ Academy of management of the Ministry of the Interior of Russia
}

\begin{abstract}
Annotation. In cases where facilities subject to mandatory state protection require the modernization or increase of the reliability / efficiency of integrated security systems (ISS), the issue of optimal reservation of their functional units (functionally necessary elements) is considered. Then it is possible to formulate the following two tasks related to the construction of the optimal ISS structure during redundancy:

1. It is required to ensure the reliability or efficiency of the ISS no less than given at the minimum cost of backup elements.

2. It is required to ensure the highest possible reliability or efficiency of the ISS at the given costs for the backup elements.

To solve the problems of optimal backup of ISS, the coordinate-wise descent method is used, which relates to search methods for solving non-linear programming problems, which allows one to obtain an approximate solution with some given accuracy. The algorithm and the program of optimum reservation of functional units of the ISS for the purpose of increase of efficiency and reliability of their functioning are offered. The coordinate descent method is based on the developed algorithm. The program operates in two modes: in the mode of optimal calculation of the cost of the ISS and in the mode of calculating the necessary efficiency of the ISS with minimal reservation costs. The program allows you to load and process more than $2^{15}$ lines (elements) of source data. Keywords: efficiency, reliability, optimal redundancy, functional unit, integrated security system.
\end{abstract}

Durdenko Vladimir A. - Doctor of Technical Sciences, Professor, Department of information technology management, Voronezh State University.

E-mail: dva_viis@mail.ru

Algazinov Edward K. - Doctor of Physical and Mathematical Sciences, Professor, Dean of the Faculty of Computer Science, Voronezh State University.

E-mail: algazinov@sc.vsu.ru

Rogozhin Alexander A. - Candidate of Technical Sciences, Deputy head, Department of educational process organization, Krasnodar university of the Ministry of the Interior of Russia.

E-mail: raa_tsbs@list.ru.

Bogdanov Dmitry S. - cadet, Krasnodar university of the Ministry of the Interior of Russia. E-mail: bdkrdu@yandex.ru.

Batorov Bator O. - postgraduate student, Department of information technology, Academy of management of the Ministry of the Interior of Russia.

E-mail: 03bator@rambler.ru. 